\title{
BLOCKWISE ZERO MAPPING IMAGE CODING
}

\author{
Zhihai He, Tian-Hu Yu, and S. K. Mitra \\ Department of Eletrical and Computer Engineering \\ University of California, Santa Barbara, CA 93106 \\ \{zhihai, yu, mitra\}@iplab.ece.ucsb.edu
}

\begin{abstract}
Uniformly quantized wavelet coefficients are first partitioned into multiscale blocks. Each block is classified either as an all-zero block or a non-zero block. A new method called zero mapping is proposed to encode all the non-zero blocks, whose output is further compressed by a first order adaptive arithmetic coder. With very low addressing and computational complexity, the proposed algorithm compares favorably with other coding algorithms reported in the literature, especially for images with considerable high frequency components.
\end{abstract}

\section{INTRODUCTION}

The wavelet transform has the ability to decorrelate an image both in space and frequency domain, compactly distributing energy into a few low frequency and a few high frequency wavelet coefficients [1]. However, some spatially varying inter-subband energy dependence still exists in the decomposed image. Many coding algorithms have been proposed in the literature $[2,3]$ to explore this inter-subband energy dependence. In the Embedded Zero-tree Wavelet (EZW) [2] and the Set Partitioning in Hierarchical Trees (SPIHT) [3] coding algorithms, an across-subband spatial orientation tree is employed to efficiently predict the significance of the wavelet coefficients. Although the SPIHT algorithm has very good rate-distortion performance, its addressing complexity, memory usage and implementation cost are relatively high.

The stack-run (SR) coding method proposed in [4] is a very efficient pre-entropy encoding scheme. After uniform quantization, the wavelet coefficients are arranged into an intermediate 1-D array in raster scan order. The consecutive zeros between any two non-zero coefficients are represented by their run-length. Each non-zero coefficient is coded by its binary representation with symbols ' 0 ' and ' 1 ', while each ZRL number is coded by its binary representation with symbols '-' and '+'. Some other tricky techniques are also employed to reduce the total length of the binary representation. Despite its very low addressing and implementation complexity, the SR coding algorithm performs better than the EZW and is competitive with the SPIHT. But its coding performance degrades at relatively high bit rates, especially when applied to some images with a lot of details, such as Barbara.

In this paper, we propose a new coding algorithm which also has low addressing and implementation complexity. But it is much more efficient than the SR coding algorithm. To this end, we first partition the image subbands into multiscale blocks. Each block is then classified either as an all-zero block or a non-zero block. Only the non-zero blocks are coded by a new method called zero mapping. In this coding algorithm, bits reduction is accomplished by the blockwise partitioning and binary classification. The compression performance of the arithmetic coder is improved by the zero mapping method.

\section{MULTISCALE BLOCKWISE PARTITIONING}

In general, after wavelet transform and quantization, there are a large number of zeros in the high frequency subbands. The encoder, especially the pre-entropy encoder should be able to code the position of zeros efficiently. Suppose we have a $\mathrm{N} \times \mathrm{N}$ block of zeros in a subband. In the EZW and SPIHT algorithm, it is encoded by the significance information of its parent(s) in the spatial tree structure. In the SR coding algorithm, it is encoded by the proceeding ZRL numbers in the raster scan order. In order to encode this allzero block more efficiently, we proposed a multiscale blockwise partitioning method. Each image subband is equally partitioned into square blocks. A block is called an all-zero block if each coefficient inside is zero. Otherwise it is called a non-zero block. Each all-zero block and non-zero block are marked by ' 0 ' and ' 1 ', respectively as shown in Fig. 1.

Due to the multiscale nature of the wavelet decom- 


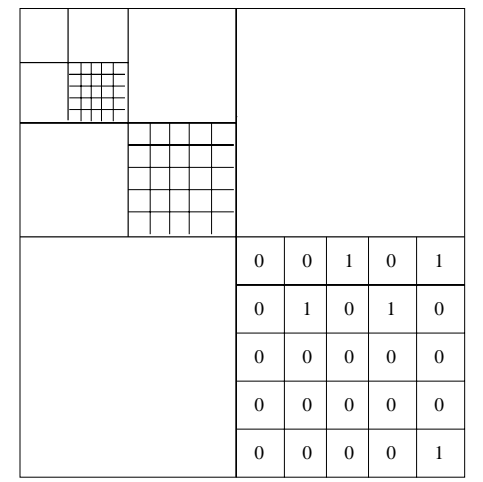

Figure 1: The multiscale blockwise partitioning.

position, different block sizes are used at different decomposition level. For example, at the first layer a block size of 16 is used; at the second layer a block size of 8 is used. The block size is halved when the decomposition level increases by one. Certainly, the block size can be freely chosen provided that the decoder have the block sizes information. If a bigger block size is chosen, more zeros in the all-zero block are encoded by the one-bit block mark ' 0 '. However, there will be more zeros included in the non-zero blocks. If a smaller block size is chosen, there will be much fewer zeros in the non-zero blocks. But more bits are needed to encode the block marks. Therefore, there is a trade-off in the choice of the block sizes.

In this paper, a five-level dyadic decomposition structure is employed. Since there are very few zeros at the fifth level, there is no need to apply the blockwise partitioning to the four subbands at this level. The block marks in each subband are scanned row by row. The block marks for all the subbands are arranged in the order from the lowest frequency to the highest frequency subband. This 1-D array of block marks are encoded by a first-order arithmetic coder.

\section{ZERO MAPPING PRE-ENTROPY CODING}

To encode the non-zero blocks, we arrange them into an 1-D intermediate array $\mathcal{L}$ in raster scan order. It is observed that there are still a large amount of zeros inside $\mathcal{L}$. Certainly, the SR coding method [4] which has very low complexity can be employed to encode $\mathcal{L}$. Two observations can be made on its coding efficiency for the 1-D array $\mathcal{L}$.

First, it is observed that some spatially varying intra- and inter-subband energy (or the amplitude of the wavelet coefficient) nonlinear correlation still exits in the decomposed image. After blockwise partitioning, all the non-zero blocks are put together, the local correlation is enhanced. For example, in the highest frequency subband, most of the non-zero wavelet coefficients have very small amplitudes. If partitioned with small blocks, these coefficients are put together much closer in $\mathcal{L}$, presenting stronger local correlation in amplitudes. But in the SR coding, the local correlation is destroyed by the binary representation of the non-zero coefficients.

Second, in the SR coding algorithm, each ZRL number is encoded by symbols from the alphabet $\left\{{ }^{\prime}-',{ }^{\prime}+'\right\}$ while each non-zero coefficient is encoded by symbols from the alphabet $\left\{{ }^{\prime} 0\right.$ ', ' 1 ' $\}$. In the output symbol stream, the codewords for the ZRL numbers and the non-zero coefficients appear alternatively with varying lengths. Therefore, it is hard for the adaptive arithmetic coder which follows the SR pre-entropy coder to build a good probability model for the symbols in the input data [5].

Based on the above analysis, to achieve better compression performance we propose the following method which is called zero mapping. First, the consecutive zeros in $\mathcal{L}$ is replaced by only one zero. The resulting 1-D array is denoted by $\mathcal{L}_{z}$ Their ZRL number is recorded separately in another sequence call $Z R L$ sequence. Both the $\mathcal{L}_{z}$ and the ZRL sequence are encoded by the variable length integer coding method [6]. For each number $q$ in these two sequences, we define its size $S$ as

$$
S=\left\{\begin{array}{ccc}
0 & \text { if } & q=0 \\
\left\lfloor\log _{2}|q|\right\rfloor+1 & \text { if } & q \neq 0 .
\end{array}\right.
$$

If $q \neq 0$, its residue bits consists of one bit for its sign and $S-1$ bits for the binary representation of $|q|-2^{S-1}$. Note that all the numbers in the ZRL sequence are positive. Therefore, there is no sign bit in the residue bits of this sequence. The size numbers are compressed further by the arithmetic coder. The residue bits are sent out directly for the sake of low computational complexity. The proposed coding algorithm is illustrated in Fig. 2.

\section{EXPERIMENTAL RESULTS}

The proposed blockwise zero mapping coding algorithm is applied many images across a wide range of bit rates. Compression results of the SPIHT, SR, EZW and the proposed algorithm for $512 \times 512$ gray images Lena and Barbara are summarized in Table 1 . The rate-distortion curves are plotted in Fig. 3. The reconstructed images of Lena at different bit rates are shown in Fig. 4. The $9 / 7$ wavelet filter, the five-level dyadic subband decomposition structure [1] and the uniform quantizer with a dead zone are employed in this work. The experimental results show that the proposed coding algorithm compares favorably with the SPIHT. For the Lena image, 


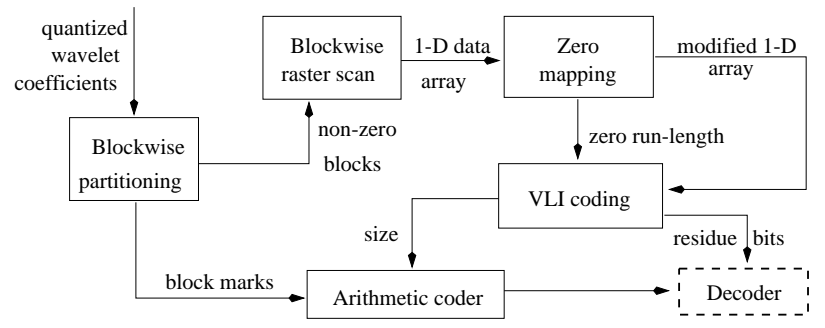

Figure 2: The proposed blockwise zero mapping coding algorithm.

the SPIHT is $0.05-0.10 \mathrm{~dB}$ better; but for the Barbara image which is regarded as difficult to code due to a lot of high frequency components, the proposed algorithm consistently outperforms the SPIHT, especially at low bit rates. When compared with the SR coding algorithm which has almost the same low complexity, the proposed algorithm consistently outperforms it by 0.3 $-0.4 \mathrm{~dB}$ for the Lena image, and $0.3-0.7 \mathrm{~dB}$ for the Barbara image. Moreover, the difference of the peak signal-to-noise ratio (PSNR) becomes bigger at higher bit rates.

\section{CONCLUDING REMARKS}

A new coding method using blockwise partitioning of subbands and zero mapping coding of non-zero blocks has been proposed. Blockwise partitioning saves bits when coding the regions of zeros. The method of zero mapping improves the performance of the adaptive arithmetic coder for the output symbol stream from the preentropy encoder. The experimental results show that the proposed coding algorithm has high compression efficiency.

It can be seen that the proposed coding algorithm is conceptually simple and has low computational complexity and memory usage. To do blockwise partitioning of one subband, we only need to know if a coefficient is zero or non-zero. In addition, the partitioning of each subband is independent from each other and no across-subband operation is involved. Furthermore, the information of the wavelet coefficients are "decomposed" into several parts with decreasing importance: the block marks, the ZRL numbers, the size numbers and the residue bits. This unique coding mechanism provides a suitable framework to perform the unequal error protection of the information bits when trans- mitting the image data through a noisy channel. For example, the critical bits such as the block marks can be protected heavily so that no transmission errors are made.

\section{Acknowledgments}

This work was supported by a University of California MICRO grant with matching support from Lucent Technologies, National Semiconductor, Tektronix Corp., and Xerox Corp..

\section{REFERENCES}

[1] M. Antonini, M. Barlaud, P. Mathieu, and I. Daubechies, "Image coding using wavelet transform," IEEE Trans. Image Processing, vol. 1, pp. 205-220, April 1992.

[2] J. M. Shapiro, "Embedded image coding using zerotrees of wavelet coefficients," IEEE Trans. Signal Processing, vol. 41, pp. 3445-3462, December 1993.

[3] A. Said and W. A. Pearlman, "A new fast and efficient image codec based on set partitioning in hierarchical trees," IEEE Trans. Circuits and System on Video Technology, vol. 6, pp. 243-250, June 1996.

[4] M. J. Tsai, J. D. Villasenor, and F. Chen, "Stackrun image coding," IEEE Trans. Circuits and System on Video Technology, vol. 6, pp. 519-521, Oct. 1996.

[5] I. H. Witten, R.M. Neal, and J.G. Cleary, "Arithmetic coding for data compression," Commun. $A C M$, vol. 30, pp. 520-540, June 1996.

[6] G. K. Wallace, "The JPEG stil picture compression standard," Commun. ACM, vol. 34, pp. 30-44, April 1991. 
Table 1: The experimental results for the Lena image.

\begin{tabular}{|c||c|c|c|c|}
\hline \multicolumn{1}{|c||}{$\begin{array}{c}\text { Bit } \\
\text { rate }\end{array}$} & \multicolumn{4}{c|}{ PSNR (dB) } \\
\cline { 2 - 5 } & SPIHT & SR & EZW & This work \\
\hline \hline 0.20 & 33.15 & 32.68 & $/$ & 33.07 \\
\hline 0.25 & 34.11 & 33.63 & 33.17 & 34.06 \\
\hline 0.30 & 34.95 & 34.48 & $/$ & 34.88 \\
\hline 0.40 & 36.24 & 35.78 & $/$ & 36.15 \\
\hline 0.50 & 37.21 & 36.79 & 36.28 & 37.10 \\
\hline 0.60 & 38.01 & 37.62 & $/$ & 37.92 \\
\hline 0.70 & 38.73 & 38.30 & $/$ & 38.61 \\
\hline 0.80 & 39.33 & 38.91 & $/$ & 39.23 \\
\hline 0.90 & 39.87 & 39.46 & $/$ & 39.75 \\
\hline 1.00 & 40.41 & 39.97 & 39.55 & 40.28 \\
\hline
\end{tabular}

Table 2: The experimental results for the Barbara image.

\begin{tabular}{|c||c|c|c|c|}
\hline \multicolumn{1}{|c||}{\multirow{2}{*}{$\begin{array}{c}\text { Bit } \\
\text { rate }\end{array}$}} & \multicolumn{4}{c|}{ PSNR (dB) } \\
\cline { 2 - 5 } & SPIHT & SR & EZW & This work \\
\hline \hline 0.20 & 26.66 & 26.55 & $/$ & 26.87 \\
\hline 0.25 & 27.58 & 27.50 & 26.77 & 27.85 \\
\hline 0.30 & 28.56 & 28.37 & $/$ & 28.75 \\
\hline 0.40 & 30.10 & 29.82 & $/$ & 30.24 \\
\hline 0.50 & 31.40 & 31.05 & 30.53 & 31.53 \\
\hline 0.60 & 32.53 & 32.18 & $/$ & 32.72 \\
\hline 0.70 & 33.75 & 33.24 & $/$ & 33.76 \\
\hline 0.80 & 34.66 & 34.14 & $/$ & 34.71 \\
\hline 0.90 & 35.60 & 34.99 & $/$ & 35.60 \\
\hline 1.00 & 36.41 & 35.76 & 35.14 & 36.43 \\
\hline
\end{tabular}
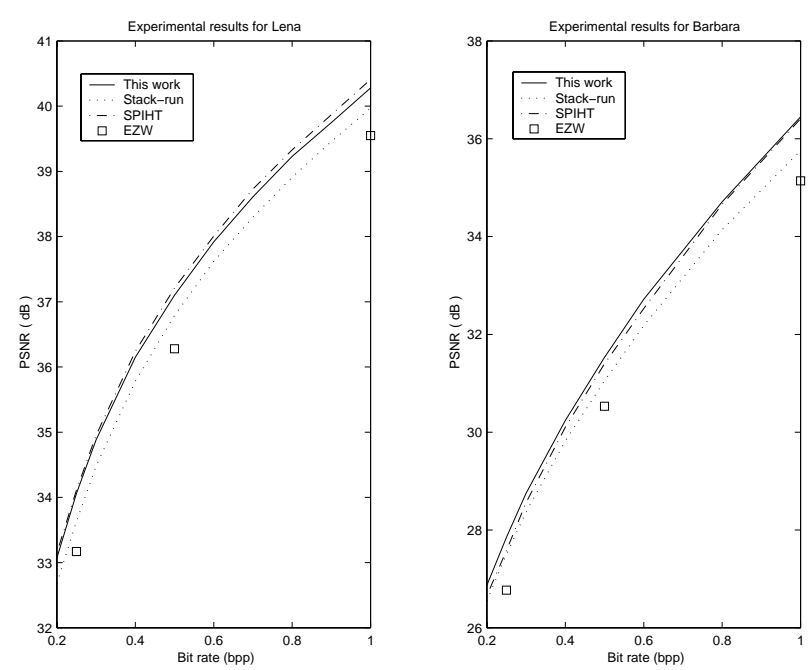

Figure 3: Experimental results for images Lena and Barbara.

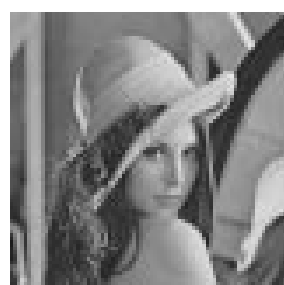

(a)

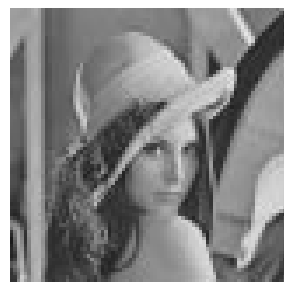

(c)

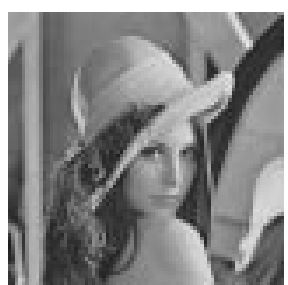

(b)

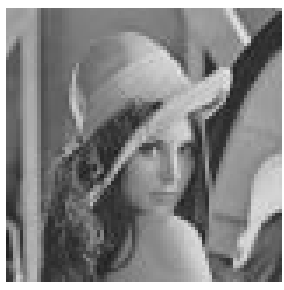

(d)
Figure 4: The performance of the propose coding algorithm on Lena: (a) the original Lena image, (b) rate $=0.20 \mathrm{bpp}$, PSNR $=33.07 \mathrm{~dB},(\mathrm{c})$ rate $=0.25 \mathrm{bpp}$, $\mathrm{PSNR}=34.06 \mathrm{~dB},(\mathrm{~d})$ rate $=0.50 \mathrm{bpp}, \mathrm{PSNR}=37.10$ dB. 